\title{
The Maxey-Riley Equation: Existence, Uniqueness and Regularity of Solutions
}

\author{
Mohammad Farazmand ${ }^{1,2 *}$ George Haller $^{2}$ \\ ${ }^{1}$ Department of Mathematics, ETH Zurich, Rämistrasse 101, 8092 Zurich Switzerland \\ ${ }^{2}$ Institute of Mechanical Systems, ETH Zurich, Tannenstrasse 3, 8092 Zurich, Switzerland
}

\begin{abstract}
The Maxey-Riley equation describes the motion of an inertial (i.e., finite-size) spherical particle in an ambient fluid flow. The equation is a second-order, implicit integro-differential equation with a singular kernel, and with a forcing term that blows up at the initial time. Despite the widespread use of the equation in applications, the basic properties of its solutions have remained unexplored. Here we fill this gap by proving local existence and uniqueness of mild solutions. For certain initial velocities between the particle and the fluid, the results extend to strong solutions. We also prove continuous differentiability of the mild and strong solutions with respect to their initial conditions. This justifies the search for coherent structures in inertial flows using the Cauchy-Green strain tensor.
\end{abstract}

\section{Introduction}

The Maxey-Riley equation [1-3] describes the motion of a small but finite-sized rigid sphere through a fluid. The equation is widely used to study the motion of a finite-size (or inertial) particle immersed in a non-uniform fluid. The behavior of such particles is of interest in various environmental and engineering problems, e.g., clustering of garbage patches in the oceans [4] and dispersion of airborne pollutants [5].

A first attempt to derive the equation of motion of an inertial particle in a non-uniform flow appears in [1]. Tchen [1] wrote the Basset-Boussinesq-Oseen equation (governing the motion of a small spherical particle in a quiescent fluid [6-8]) in a frame co-moving with a fluid parcel in an unsteady flow, accounting for various forces that arise in such non-inertial frames. Later, the exact form of the forces exerted on the particle were debated and corrected by several authors [see, e.g., $2,3,9]$. Today, the most widely accepted form of the equations is the Maxey-Riley (MR) equation [3] with the corrections due to Auton et al. [9] and Maxey [10].

To recall the exact form of the MR equation, we let $u: \mathcal{D} \times \mathbb{R}^{+} \rightarrow \mathbb{R}^{n}$ denote the velocity field describing the flow of a fluid in an open spatial domain $\mathcal{D} \subseteq \mathbb{R}^{n}$, where $n=2$ or $n=3$ for twoor three-dimensional flows, respectively. A fluid trajectory is then the solution of the differential equation $\dot{x}=u(x, t)$ with some initial condition $x\left(t_{0}\right)=x_{0}$. A spherical inertial particle, however,

\footnotetext{
*Corresponding author; email address: farazmam@ethz.ch
} 
follows a different trajectory $y(t) \in \mathcal{D}$, which satisfies the MR equation

$$
\begin{aligned}
\ddot{y}= & \frac{R}{2} \frac{D}{D t}\left(3 u(y, t)+\frac{\gamma}{10} \mu^{-1} \Delta u(y, t)\right)+\left(1-\frac{3 R}{2}\right) g \\
& -\mu\left(\dot{y}-u(y, t)-\frac{\gamma}{6} \mu^{-1} \Delta u(y, t)\right) \\
& -\kappa \mu^{1 / 2}\left\{\int_{t_{0}}^{t} \frac{\dot{w}(s)}{\sqrt{t-s}} \mathrm{~d} s+\frac{w\left(t_{0}\right)}{\sqrt{t-t_{0}}}\right\},
\end{aligned}
$$

where

$$
w(t)=\dot{y}(t)-u(y(t), t)-\frac{\gamma}{6} \mu^{-1} \Delta u(y(t), t) .
$$

The initial conditions for the inertial particle are given as $y\left(t_{0}\right)=y_{0}$ and $\dot{y}\left(t_{0}\right)=v_{0}$. The material derivative $\frac{D}{D t} \doteq \partial_{t}+u \cdot \nabla$ denotes the time derivative along a fluid trajectory.

All the variables and parameters in equations (1) and (2) are dimensionless, nondimensionalized by characteristic length scale $L$, characteristic velocity $U$ and characteristic time scale $T=L / U$ of the fluid flow. The dimensionless parameters are

$$
R=\frac{2 \rho_{f}}{\rho_{f}+2 \rho_{p}}, \mu=\frac{R}{S t}, \kappa=\sqrt{\frac{9 R}{2 \pi}}, \gamma=\frac{9 R}{2 \mathrm{Re}},
$$

where $\rho_{f}$ and $\rho_{p}$ are the density of the fluid and the particle, respectively. The constant, dimensionless vector of gravity is denoted by $g$. The Stokes (St) and Reynolds (Re) numbers are defined as

$$
\text { St }=\frac{2}{9}\left(\frac{a}{L}\right)^{2} \operatorname{Re}, \quad \operatorname{Re}=\frac{U L}{\nu},
$$

where $a$ is the radius of the particle and $\nu$ denotes the kinematic viscosity of the fluid.

Equation (1) is a system of nonlinear, fractional-order differential equations. The fractional order is due to the memory term

$$
\frac{\mathrm{d}}{\mathrm{d} t} \int_{t_{0}}^{t} \frac{w(s)}{\sqrt{t-s}} \mathrm{~d} s=\int_{t_{0}}^{t} \frac{\dot{w}(s)}{\sqrt{t-s}} \mathrm{~d} s+\frac{w\left(t_{0}\right)}{\sqrt{t-t_{0}}}
$$

where the identity is obtained by subsequent differentiation and integration-by-part [see, e.g., 11]. The memory term is a fractional derivative of order $1 / 2$ in the Riemann-Liouville sense [11, 12]. Physically, it represents the Basset-Boussinesq force $[6,7,13]$ resulting from the lagging boundary layer development around the particle, as it moves through the fluid [3].

In the original derivation of the MR equation [3], it is implicitly assumed that the initial velocity of the particle $v_{0}$ is such that $w\left(t_{0}\right)=0$ holds. Equation (1), however, is the most general form of the MR equation which was derived later [10] and allows for a general initial particle velocity $v_{0}$.

Without the memory term and for $w\left(t_{0}\right)=0$, the MR equation is an ordinary differential equation, whose solutions are well known to be regular for any smooth ambient velocity field $u(x, t)$. The memory term, however, introduces complications in the analysis and numerical solution of the equation. It contains an implicit term through the integral with an integrand depending on the particle acceleration $\ddot{y}$. Because of its implicit nature, it is not a priori clear if the MR differential equation defines a dynamical system, i.e., a process with a well-defined flow map.

Furthermore, when nonzero, the unbounded term $w\left(t_{0}\right) / \sqrt{t-t_{0}}$ further complicates equation (1), imparting an instantaneously infinite force at the initial time. This term is often ignored for convenience, even though its omission imposes a special constraint on the initial particle velocity that is hard to justify physically [10].

For the above reasons, the memory term has routinely been neglected in studies of inertial particle dynamics (see, e.g., Maxey [14], Babiano et al. [15], Haller and Sapsis [16]), until recent 
studies demonstrated convincingly the quantitative and qualitative importance of the memory term (see, e.g., [17-19] for experimental and [20-22] for numerical studies.).

In addition to theoretical difficulties, the memory term also complicates the numerical treatment of the full MR equation. This equation is certainly not solvable with standard numerical schemes such as Runge-Kutta algorithms. To this end, involved schemes have been developed for numerical treatment of the memory term (see Daitche [12] and references therein).

All these numerical schemes implicitly assume the existence and uniqueness of solutions of the MR equation. The solutions can indeed be found explicitly for certain simple velocity fields [17, 23]. To the best of our knowledge, however, general existence and uniqueness results have not been proven, and cannot be directly concluded from existing results on broader classes of evolution equations (see [11, 24-26] for related but not applicable results on integro- and fractional-order differential equations). In the absence of such results, the existence and regularity of solutions for a nonlinear system of fractional-order differential equation, such as the MR equation, is far from obvious.

Here, we present the first proof of local existence and uniqueness of mild solutions to the full MR equation. The solutions become classical (strong) solutions to (1) for initial conditions satisfying $w\left(t_{0}\right)=0$. Moreover, we show that both the mild and the strong solutions are continuously differentiable with respect to their initial conditions. As a consequence, coherent-structure detection methods utilizing the derivative of the flow map in the absence of the memory term [27] can also be employed in the present, more general context.

We start with re-writing the MR equation as a system of differential equations (see Eq. (8) below) in terms of the particle position $y$ and the function $w$ defined in (2). Multi-dimensional reformulations of the MR equation have appeared before [12, 23, 28] but remained inaccessible to general mathematical analysis due to the implicit dependence of their right-hand sides on $\dot{y}$.

Our formulation turns the MR equation into a nonlinear system of fractional-order differential equations in terms of $y$ and $w$. The standard techniques for the proof of existence and uniqueness of solutions of such equations assume Lipschitz continuity of the right hand side with respect to the $(y, w)$ variable $[11,26]$. This assumption fails for the MR equation (see the term $M_{u}(y, t) w$ in Eq. (8) below). Therefore, as discussed in Section $\S 3$, modifications to the standard function spaces, estimates and assumptions are required.

\section{Preliminaries}

We start by letting the velocity of the inertial particle be $v: \mathbb{R}^{+} \rightarrow \mathbb{R}^{n}$, and use this notation to rewrite (1) as a first-order system of equations

$$
\begin{aligned}
\dot{y}= & v \\
\dot{v}= & R \frac{D u}{D t}+\left(1-\frac{3 R}{2}\right) g+\frac{R}{2} \frac{D}{D t}\left(u+\frac{\gamma}{10} \mu^{-1} \Delta u\right) \\
& -\mu\left(v-u-\frac{\gamma}{6} \mu^{-1} \Delta u\right) \\
& -\kappa \mu^{1 / 2} \frac{\mathrm{d}}{\mathrm{d} t} \int_{t_{0}}^{t} \frac{w(s)}{\sqrt{t-s}} \mathrm{~d} s,
\end{aligned}
$$

with the function $w(t)$ defined as in (2). The memory term is written as a fractional-order derivative through identity (5). As earlier, the material derivative $\frac{D}{D t} \doteq \partial_{t}+u \cdot \nabla$ denotes a time derivative along a fluid trajectory. Also $\frac{d}{d t} \doteq \partial_{t}+v \cdot \nabla$ denotes temporal differentiation along the inertial trajectory $y(t)$. The two derivatives are related by the identity

$$
\frac{d}{d t}=\frac{D}{D t}+(v-u) \cdot \nabla
$$

For notational simplicity, we will also use the dot symbol for the derivative $\frac{d}{d t}$. 
We rewrite (6) in the more compact form

$$
\begin{aligned}
\dot{y} & =w+A_{u}(y, t), \\
\dot{w} & =-\mu w-M_{u}(y, t) w-\kappa \mu^{1 / 2} \frac{\mathrm{d}}{\mathrm{d} t} \int_{t_{0}}^{t} \frac{w(s)}{\sqrt{t-s}} \mathrm{~d} s+B_{u}(y, t),
\end{aligned}
$$

where

$$
\begin{aligned}
A_{u}= & u+\frac{\gamma}{6} \mu^{-1} \Delta u \\
B_{u}= & \left(\frac{3 R}{2}-1\right)\left(\frac{D u}{D t}-g\right)+\left(\frac{R}{20}-\frac{1}{6}\right) \gamma \mu^{-1} \frac{D}{D t} \Delta u \\
& -\frac{\gamma}{6} \mu^{-1}\left[\nabla u+\frac{\gamma}{6} \mu^{-1} \nabla \Delta u\right] \Delta u, \\
M_{u}= & \nabla u+\frac{\gamma}{6} \mu^{-1} \nabla \Delta u,
\end{aligned}
$$

are known functions in terms of the fluid velocity $u$. The terms $A_{u}, B_{u}: \mathcal{D} \times R^{+} \rightarrow \mathbb{R}^{n}$ represent vector fields while $M_{u}: \mathcal{D} \times \mathbb{R}^{+} \rightarrow \mathbb{R}^{n \times n}$ is a tensor field. Note that equation (8) is linear in $w$ and, for a typical fluid velocity field $u$, non-linear in $y$. The corresponding initial conditions for (8) are $y\left(t_{0}\right)=y_{0}$ and $w\left(t_{0}\right)=w_{0}:=v_{0}-u\left(y_{0}, t_{0}\right)-\frac{\gamma}{6} \mu^{-1} \Delta u\left(y_{0}, t_{0}\right)$.

\section{Local existence and uniqueness}

\subsection{Approach}

This section is devoted to proving the local existence and uniqueness of solutions of (8) under certain smoothness assumptions on the fluid velocity field $u$.

Integrating equation (8) formally, one obtains

$$
\begin{aligned}
& y(t)=y_{0}+\int_{t_{0}}^{t}\left[w(s)+A_{u}(y(s), s)\right] \mathrm{d} s, \\
& w(t)=w_{0}+\int_{t_{0}}^{t}\left[-\mu w(s)-M_{u}(y(s), s) w(s)-\kappa \mu^{1 / 2} \frac{w(s)}{\sqrt{t-s}}+B_{u}(y(s), s)\right] \mathrm{d} s,
\end{aligned}
$$

where, for notational simplicity, we have omitted the dependence of $y$ and $w$ on $y_{0}$ and $w_{0}$. A mild solution of the MR equation is a function $(y(t), w(t))$ that satisfies the integral equation (9). The same solution is also a strong solution if it is smooth enough to also satisfy the differential form (8) of the MR equation.

Equation (9) can be viewed as a fixed point problem for the map

$$
(P \Phi)(t)=\left(\begin{array}{c}
y_{0}+\int_{t_{0}}^{t}\left[\eta(s)+A_{u}(\xi(s), s)\right] \mathrm{d} s \\
w_{0}+\int_{t_{0}}^{t}\left[-\left(\mu+\frac{\kappa \mu^{1 / 2}}{\sqrt{t-s}}+M_{u}(\xi(s), s)\right) \eta(s)+B_{u}(\xi(s), s)\right] \mathrm{d} s
\end{array}\right),
$$

where $\Phi=(\xi, \eta) \in \mathbb{R}^{2 n}$. We will establish the existence of mild solutions to the MR equations by showing that $P$ has a unique fixed point on an appropriate function space under general regularity assumptions on the fluid velocity $u$.

\subsection{Set-up}

We will use $|\cdot|$ to denote the Euclidean norm on $\mathbb{R}^{m}$ with $m \in\{n, 2 n\}$. The induced operator norm of a square matrix acting on $\mathbb{R}^{m}$ is denoted by $\|\cdot\|$. For continuous functions defined on $\mathbb{R}^{m}$, we denote the supremum norm by $\|\cdot\|_{\infty}$. 
Let $X_{T, K}$ denote the set of continuous functions mapping from the interval $\left[t_{0}, t_{0}+T\right]$ into $\mathbb{R}^{m}$ that are uniformly bounded by the constant $K>0$ :

$$
X_{T, K}:=\left\{f \in C\left(\left[t_{0}, t_{0}+T\right] ; \mathbb{R}^{m}\right):\|f\|_{\infty} \leq K\right\} .
$$

Since $\left(C\left(\left[t_{0}, t_{0}+T\right] ; \mathbb{R}^{m}\right),\|\cdot\|_{\infty}\right)$ is a Banach space, the space $\left(X_{T, K},\|\cdot\|_{\infty}\right)$ is a complete metric space, for $X_{T, K}$ is a closed subset of $C\left(\left[t_{0}, t_{0}+T\right] ; \mathbb{R}^{m}\right)$.

First, we would like to show that $P$ defined in (10) maps $X_{T, K}$ into itself. To this end, we need the following assumption.

(H1) The velocity field $u(x, t)$ is three times continuously differentiable in its arguments over the domain $\mathcal{D} \times \mathbb{R}^{+}$, and its partial derivatives (including mixed partials) are uniformly bounded and Lipschitz continuous up to order three.

\subsection{Existence and uniqueness of solutions}

Under assumption (H1), we obtain the following result:

Lemma 1. Assume that (H1) holds. Then for any $y_{0} \in \mathcal{D}$ and $w_{0} \in \mathbb{R}^{n}$, there exist $K>0$ large enough and $\delta>0$ small enough, such that, for any $T \in[0, \delta]$, we have $P: X_{T, K} \rightarrow X_{T, K}$.

Proof. Under assumption (H1), the vector fields $A_{u}, B_{u}: \mathcal{D} \times \mathbb{R}^{+} \rightarrow \mathbb{R}^{n}$ and the tensor field $M_{u}$ : $\mathcal{D} \times \mathbb{R}^{+} \rightarrow \mathbb{R}^{n \times n}$ are continuous and uniformly bounded. Specifically, there exists a constant $L_{b}>0$ such that

$$
\left\|A_{u}\right\|_{\infty},\left\|B_{u}\right\|_{\infty},\left\|M_{u}\right\|_{\infty} \leq L_{b} .
$$

Then, based on eq. (10), the quantity $P \Phi$ satisfies the estimate

$$
\begin{aligned}
|P \Phi(t)| \leq & \left\|y_{0}+\int_{t_{0}}^{t}\left[\eta(s)+A_{u}(\xi(s), s)\right] \mathrm{d} s\right\|_{\infty} \\
& +\left\|w_{0}+\int_{t_{0}}^{t}\left[\left(\mu+\frac{\kappa \mu^{1 / 2}}{\sqrt{t-s}}+M_{u}(\xi(s), s)\right) \eta(s)+B_{u}(\xi(s), s)\right] \mathrm{d} s\right\|_{\infty} \\
\leq & \left|y_{0}\right|+\left|w_{0}\right|+\|\eta\|_{\infty}\left(\left(t-t_{0}\right)+\mu\left(t-t_{0}\right)+2 \kappa \mu^{1 / 2} \sqrt{t-t_{0}}+L_{b}\left(t-t_{0}\right)\right)+2 L_{b}\left(t-t_{0}\right) \\
& \leq\left|y_{0}\right|+\left|w_{0}\right|+\|\Phi\|_{\infty}\left(\left(t-t_{0}\right)+\mu\left(t-t_{0}\right)+2 \kappa \mu^{1 / 2} \sqrt{t-t_{0}}+L_{b}\left(t-t_{0}\right)\right)+2 L_{b}\left(t-t_{0}\right) .
\end{aligned}
$$

Now take $K=4 \max \left\{\left|y_{0}\right|,\left|w_{0}\right|\right\}$ and $\delta>0$ small enough such that

$$
\delta+\mu \delta+2 \kappa \mu^{1 / 2} \sqrt{\delta}+L_{b} \delta<\frac{1}{4}, \quad 2 L_{b} \delta<\frac{K}{4} .
$$

Then, for any $T \in[0, \delta],\|P \Phi\|_{\infty} \leq K$ given that $\Phi \in X_{T, K}$. The continuity of $P \Phi:\left[t_{0}, t_{0}+T\right] \rightarrow \mathbb{R}^{2 n}$ follows from assumption (H1) after one notes that, for $\eta \in X_{T, K}$, the term $\int_{t_{0}}^{t} \frac{\eta(s)}{\sqrt{t-s}} \mathrm{~d} s$ in (10) is continuous in $t$.

We establish the existence of a unique solution to (9) by proving that $P$ is a contraction mapping on $X_{T, K}$ and hence has a unique fixed point.

Lemma 2. Assume that (H1) holds. Then there exists $\delta>0$ such that, for any $T \in[0, \delta]$ and $\Phi_{1}, \Phi_{2} \in X_{T, K}$

$$
\left\|P \Phi_{1}-P \Phi_{2}\right\|_{\infty} \leq \frac{1}{2}\left\|\Phi_{1}-\Phi_{2}\right\|_{\infty}
$$


Proof. Note that as a direct consequence of assumption (H1), the maps $A_{u}(\cdot, t), B_{u}(\cdot, t): \mathcal{D} \rightarrow \mathbb{R}^{n}$ and $M_{u}(\cdot, t): \mathcal{D} \rightarrow \mathbb{R}^{n \times n}$ are Lipschitz continuous, uniformly in time, i.e., there is a constant $L_{c}>0$ such that, for any $t \in\left[t_{0}, t_{0}+T\right]$ and $y_{1}, y_{2} \in \mathcal{D}$,

$$
\begin{gathered}
\left|A_{u}\left(y_{1}, t\right)-A_{u}\left(y_{2}, t\right)\right| \leq L_{c}\left|y_{1}-y_{2}\right|, \\
\left|B_{u}\left(y_{1}, t\right)-B_{u}\left(y_{2}, t\right)\right| \leq L_{c}\left|y_{1}-y_{2}\right|, \\
|| M_{u}\left(y_{1}, t\right)-M_{u}\left(y_{2}, t\right) \| \leq L_{c}\left|y_{1}-y_{2}\right| .
\end{gathered}
$$

Let $\Phi_{1}, \Phi_{2} \in X_{T, K}$, where $\Phi_{i}=\left(\xi_{i}, \eta_{i}\right)$. Using the above inequalities, we have

$$
\begin{aligned}
\left|\left(P \Phi_{1}\right)(t)-\left(P \Phi_{2}\right)(t)\right| \leq & \int_{t_{0}}^{t}\left(\left|\eta_{1}(s)-\eta_{2}(s)\right|+\left|A_{u}\left(\xi_{1}(s), s\right)-A_{u}\left(\xi_{2}(s), s\right)\right|\right) \mathrm{d} s+ \\
& \int_{t_{0}}^{t}\left[\mu\left|\eta_{1}(s)-\eta_{2}(s)\right|+\kappa \mu^{1 / 2} \frac{\left|\eta_{1}(s)-\eta_{2}(s)\right|}{\sqrt{t-s}}+\right. \\
& \left|M_{u}\left(\xi_{1}(s), s\right) \eta_{1}(s)-M_{u}\left(\xi_{2}(s), s\right) \eta_{2}(s)\right|+ \\
& \left.\left|B_{u}\left(\xi_{1}(s), s\right)-B_{u}\left(\xi_{2}(s), s\right)\right|\right] \mathrm{d} s \\
\leq & {\left[\left(t-t_{0}\right)+\mu\left(t-t_{0}\right)+2 \kappa \mu^{1 / 2} \sqrt{t-t_{0}}\right]\left\|\eta_{1}-\eta_{2}\right\|_{\infty}+} \\
& 2 L_{c}\left(t-t_{0}\right)\left\|\xi_{1}-\xi_{2}\right\|_{\infty}+ \\
& \int_{t_{0}}^{t}\left[\left|M_{u}\left(\xi_{1}(s), s\right) \eta_{1}(s)-M_{u}\left(\xi_{1}(s), s\right) \eta_{2}(s)\right|+\right. \\
\leq & {\left[\left(M_{u}\left(\xi_{1}(s), s\right) \eta_{2}(s)-M_{u}\left(\xi_{2}(s), s\right) \eta_{2}(s) \mid\right] \mathrm{d} s\right.} \\
& \left(2+K\left(t-t_{0}\right)+2 \kappa \mu^{1 / 2} \sqrt{t-t_{0}}+L_{b}\left(t-t_{0}\right)\right]\left\|\eta_{1}-\eta_{2}\right\|_{\infty}+
\end{aligned}
$$

where we used the fact that

$$
\begin{gathered}
\int_{t_{0}}^{t}\left|M_{u}\left(\xi_{1}(s), s\right) \eta_{1}(s)-M_{u}\left(\xi_{2}(s), s\right) \eta_{2}(s)\right| \mathrm{d} s= \\
\int_{t_{0}}^{t}\left|M_{u}\left(\xi_{1}(s), s\right)\left(\eta_{1}(s)-\eta_{2}(s)\right)+\left(M_{u}\left(\xi_{1}(s), s\right)-M_{u}\left(\xi_{2}(s), s\right)\right) \eta_{2}(s)\right| \mathrm{d} s \\
\leq \int_{t_{0}}^{t}\left\|M_{u}\left(\xi_{1}(s), s\right)\right\|\left|\eta_{1}(s)-\eta_{2}(s)\right| \mathrm{d} s+\int_{t_{0}}^{t}\left\|M_{u}\left(\xi_{1}(s), s\right)-M_{u}\left(\xi_{2}(s), s\right)\right\|\left|\eta_{2}(s)\right| \mathrm{d} s \\
\leq\left(t-t_{0}\right) L_{b}\left\|\eta_{1}-\eta_{2}\right\|_{\infty}+\left(t-t_{0}\right) L_{c}\left\|\eta_{2}\right\|_{\infty}\left\|\xi_{1}-\xi_{2}\right\|_{\infty} .
\end{gathered}
$$

Therefore, one can take $\delta>0$ small enough such that, for any $t \in\left[t_{0}, t_{0}+\delta\right]$,

$$
\begin{aligned}
\left|\left(P \Phi_{1}\right)(t)-\left(P \Phi_{2}\right)(t)\right| & \leq \frac{1}{4}\left(\left\|\xi_{1}-\xi_{2}\right\|_{\infty}+\left\|\eta_{1}-\eta_{2}\right\|_{\infty}\right) \\
& \leq \frac{1}{2}\left\|\Phi_{1}-\Phi_{2}\right\|_{\infty} .
\end{aligned}
$$

Here, for the last inequality, we have used the fact that $\|\xi\|_{\infty}+\|\eta\|_{\infty}<2\|\Phi\|_{\infty}$. Hence, we obtain the contraction property

$$
\left\|P \Phi_{1}-P \Phi_{2}\right\|_{\infty} \leq \frac{1}{2}\left\|\Phi_{1}-\Phi_{2}\right\|_{\infty},
$$

as claimed.

Lemma 2 leads to our main existence result. 
Theorem 1. [Local existence of mild solutions] Assume that (H1) holds. Then for any initial condition $\left(y_{0}, w_{0}\right) \in \mathcal{D} \times \mathbb{R}^{n}$, there exists $\delta>0$ such that over the time interval $\left[t_{0}, t_{0}+\delta\right]$, the integral equation (9) has a unique solution $(y(t), w(t))$ with $\left(y\left(t_{0}\right), w\left(t_{0}\right)\right)=\left(y_{0}, w_{0}\right)$. As consequence, the function $y(t)$ is a mild solution of the original form (1) of the Maxey-Riley equation.

Proof. By Lemma 2, for any $y_{0} \in \mathcal{D}$ and $w_{0} \in \mathbb{R}^{n}$, there exist $K>0$ and $\delta>0$ such that for any $T \in[0, \delta]$, the map $P: X_{T, K} \rightarrow X_{T, K}$ is a contraction on the complete metric space $X_{T, K}$. As a consequence, the mapping $P$ has a unique fixed point $(y, w):\left[t_{0}, t_{0}+\delta\right] \rightarrow \mathcal{D} \times \mathbb{R}^{n}$. By the definition of $P$, this fixed point solves the integral equation (9), and hence is a mild solution of (8), or equivalently, of (1).

Remark 1. The solution $y(t)$ is, in general, not a strong solution of (1), because it is only once continuously differentiable at $t=t_{0}$, and hence only satisfies the integrated form of $w(t)$. The following example demonstrates the lack of existence of strong solutions in a simple case where the $w$-equation in (8) can be solved explicitly.

Example 1. For a uniform fluid at rest (i.e., $u \equiv 0$ ), if we neglect the effect of gravity (i.e., set $g=0$ ), $A_{u}, B_{u}$ and $M_{u}$ in equation (8) vanish. Then, the equation for $w$ reduces to

$$
\dot{w}+\kappa \mu^{1 / 2} \frac{\mathrm{d}}{\mathrm{d} t} \int_{0}^{t} \frac{w(s)}{\sqrt{t-s}} \mathrm{~d} s+\mu w=0,
$$

with the initial time $t_{0}=0$ and an arbitrary initial condition $w(0)=w_{0}$. Taking the Laplace transform of this equation, we obtain

$$
W(p)=\frac{1}{p+G p^{1 / 2}+\mu} w_{0},
$$

where $G=\sqrt{9 R \mu / 2}$ and $W$ denotes the Laplace transform of $w$. For $R<8 / 9$, the inverse Laplace transform yields the exact solution

$$
w(t)=w_{0}\left\{\mathrm{e}^{-\alpha t} \cos (\beta t)+\frac{G^{2}}{2 \beta} \mathrm{e}^{-\alpha t} \sin (\beta t)-\frac{G}{\sqrt{\pi}} \int_{0}^{t} \frac{\mathrm{e}^{-\alpha s} \cos (\beta s)-(\alpha / \beta) \mathrm{e}^{-\alpha s} \sin (\beta s)}{\sqrt{t-s}} \mathrm{~d} s\right\},
$$

with $\alpha=\mu(1-9 R / 4)$ and $\beta=G \sqrt{\mu(1-9 R / 8)}$. Defining

$$
c(s)=\mathrm{e}^{-\alpha s} \cos (\beta s)-(\alpha / \beta) \mathrm{e}^{-\alpha s} \sin (\beta s),
$$

and taking the derivative of $w$ with respect to time $t$, we obtain

$$
\dot{w}(t)=w_{0}\left\{\left(\frac{G^{2}}{2}-\alpha\right) \mathrm{e}^{-\alpha t} \cos (\beta t)-\left(\frac{\alpha G^{2}}{2 \beta}+\beta\right) \mathrm{e}^{-\alpha t} \sin (\beta t)-\frac{G}{\sqrt{\pi}} \int_{0}^{t} \frac{\dot{c}(s)}{\sqrt{t-s}} \mathrm{~d} s-\frac{G}{\sqrt{\pi t}}\right\} .
$$

For any $T>0$, the first three terms in $\dot{w}$ are continuous over the time interval $[0, T]$. The last term $\frac{G}{\sqrt{\pi t}}$, however, is discontinuous at $t=0$. This concludes our example showing that, in general, the MR equation with non-zero initial condition $w_{0}$ only admits mild solutions.

As mentioned in the Introduction, the original form of the MR equation [3] assumes the initial velocity $w\left(t_{0}\right)=0$. This assumption is mathematically convenient, as it removes the unbounded term from (1). Physically, however, the assumption is artificial, and cannot be enforced at the release of an inertial particle.

Nevertheless, $w\left(t_{0}\right)=0$ has been routinely assumed in various studies of the MR equation (see, e.g., Babiano et al. [15], Candelier et al. [17], Daitche and Tél [20]) as an important special case. We now show that under this special assumption, the MR equation in fact has strong solutions.

Theorem 2. Assume that (H1) holds. Then for any $y_{0} \in \mathcal{D}$, there exists $\delta>0$ such that, over the time interval $\left[t_{0}, t_{0}+\delta\right]$, the Maxey-Riley equation (1) has a unique solution satisfying $y\left(t_{0}\right)=y_{0}$ and $w\left(t_{0}\right)=0$.

Proof. See Appendix A. 


\subsection{Regularity of solutions}

Here we show the differentiability of the solutions of (8) with respect to the initial condition $\left(y_{0}, w_{0}\right)$. Assume that a solution $(y(t), w(t))$ is differentiable at $\left(y_{0}, w_{0}\right)$ and denote the derivative of $y$ and $w$ with respect to $\left(y_{0}, w_{0}\right)$ by $D y$ and $D w$, respectively.

Differentiating (8) formally and integrating in time, we obtain that $D y, D w: \mathbb{R}^{+} \rightarrow \mathbb{R}^{n \times 2 n}$ must satisfy

$$
\begin{gathered}
D y(t)=\left(I_{n} \mid O_{n}\right)+\int_{t_{0}}^{t}\left[D w(s)+\nabla A_{u}(y(s), s) D y(s)\right] \mathrm{d} s \\
\begin{aligned}
D w(t)=\left(O_{n} \mid I_{n}\right)+\int_{t_{0}}^{t}[ & -\mu D w(s)-\mathcal{L}(y(s), w(s), s) D y(s)-M_{u}(y(s), s) D w(s) \\
& \left.\quad-\kappa \mu^{1 / 2} \frac{D w(s)}{\sqrt{t-s}}+\nabla B_{u}(y(s), s) D y(s)\right] \mathrm{d} s,
\end{aligned}
\end{gathered}
$$

where $\mathcal{L}$ denotes the $n \times n$ matrix given by

$$
\mathcal{L}_{i j}(y(s), w(s), s)=\left.\sum_{k} \frac{\partial M_{i k}}{\partial y_{j}}\right|_{(y(s), s)} w_{k}(s) .
$$

The matrices $I_{n}$ and $O_{n}$ denote the identity and null matrices on $\mathbb{R}^{n \times n}$.

The differentiability of the solution $(y, w)$ with respect to the initial condition $\left(y_{0}, w_{0}\right)$, therefore, is equivalent to the existence and uniqueness of solutions to the equations (13). We show that under further regularity assumptions on the fluid velocity $u$, a unique solution to these equations does exist. In particular, we need the following assumption:

(H2) The velocity field $u(x, t)$ is four times continuously differentiable in its arguments over the domain $\mathcal{D} \times \mathbb{R}^{+}$. Its partial derivatives (including mixed partials) are uniformly bounded and Lipschitz continuous up to order three.

Theorem 3. Assume that (H2) holds. Then for any $y_{0} \in \mathcal{D}$ and $w_{0} \in \mathbb{R}^{n}$, there exists $\delta>0$ small enough such that, a unique mild solution $(y(t), w(t))$ of (8) exists over the time interval $\left[t_{0}, t_{0}+\delta\right]$, and is continuously differentiable with respect to its initial condition $\left(y_{0}, w_{0}\right)$.

Proof. Note that the map $\mathcal{P}$ defined by the right hand side of (13) is linear in $D y$ and $D w$. It follows from assumption (H2) that $\mathcal{P}$ maps $C\left(\left[t_{0}, t_{0}+\delta\right] ; \mathbb{R}^{2 n \times 2 n}\right)$ into itself for any $\delta \in \mathbb{R}^{+}$. Furthermore, for $\delta>0$ small enough, the map $\mathcal{P}$ is a contraction $C\left(\left[t_{0}, t_{0}+\delta\right] ; \mathbb{R}^{2 n \times 2 n}\right)$ by an argument similar to Lemma (2) (omitted here for brevity). Therefore, there are unique derivatives $D y, D w:\left[t_{0}, t_{0}+\delta\right] \rightarrow$ $\mathbb{R}^{n \times 2 n}$ that belong to the function space $C\left(\left[t_{0}, t_{0}+\delta\right] ; \mathbb{R}^{n \times 2 n}\right)$ and solve equations (13).

Remark 2. For the special case $w\left(t_{0}\right)=0$, one can similarly show that the strong solution $(y(t), w(t))$ is differentiable with respect to the initial position $y_{0}$.

\section{Conclusion}

We have proved the local existence and uniqueness of solutions of the Maxey-Riley (MR) equation. In the most general case, the solutions exist only in a weak sense. This is consistent with the physics of the problem, because an initial velocity mismatch between the ambient fluid and the particle creates a vorticity layer around the particle with high drag. This drag force is modeled in the MR equation by a term proportional to $1 / \sqrt{t-t_{0}}$, which is singular but integrable. As a result, the solution of the MR equation is continuous but only differentiable for $t>t_{0}$.

In theoretical and numerical investigations of the MR equation, it is routinely assumed that the relative velocity term $w(t)$ is chosen in a way that eliminates the infinitely large force at time 
$t=t_{0}$. We have shown that under this assumption, a unique strong solution exists to the MR equation. Moreover, both the mild and the strong solutions are differentiable with respect to their initial conditions.

Remaining challenges for the MR equations include global existence and uniqueness and an asymptotic analysis of the solutions, at least for small inertial particles.

\section{Acknowledgment}

We are grateful to Tibor Krisztin for his insights on the Maxey-Riley equations, and for pointing out Ref. [24]. We would also like to acknowledge useful conversations with Martin Maxey and Tamás Tél on the Maxey-Riley equations. We are also thankful to Daniel Karrasch for fruitful discussions on an early draft of this manuscript.

\section{Appendix A Proof of Theorem 2}

First, we slightly reformulate the MR equation. If continuously differentiable solutions to equation (8) exist, then the integral term in the equation can be re-written as

$$
\frac{\mathrm{d}}{\mathrm{d} t} \int_{t_{0}}^{t} \frac{w(s)}{\sqrt{t-s}} \mathrm{~d} s=\int_{t_{0}}^{t} \frac{\dot{w}(s)}{\sqrt{t-s}} \mathrm{~d} s
$$

since $w\left(t_{0}\right)=0$. As a consequence, the MR equation (8) can be written as

$$
\begin{aligned}
& \dot{y}=w+A_{u}(y, t), \\
& \dot{w}=-\mu w-M_{u}(y, t) w-\kappa \mu^{1 / 2} \int_{t_{0}}^{t} \frac{\dot{w}(s)}{\sqrt{t-s}} \mathrm{~d} s+B_{u}(y, t) .
\end{aligned}
$$

Now, we would like to show that this latter equation, in fact, admits continuously differentiable solutions satisfying $y\left(t_{0}\right)=y_{0}$ and and $w\left(t_{0}\right)=0$. Our proof will differ from the proof of Theorem 1. The main ideas follow those of Burton and Purnaras [25], although the details are quite different. In particular, the results of [25] do not apply in our context.

We need to show that there are unique bounded continuous functions $\phi, \psi:\left[t_{0}, t_{0}+T\right] \rightarrow \mathbb{R}^{n}$ such that the functions

$$
\begin{gathered}
y(t)=y_{0}+\int_{t_{0}}^{t} \phi(s) \mathrm{d} s, \\
w(t)=\int_{t_{0}}^{t} \psi(s) \mathrm{d} s,
\end{gathered}
$$

solve equation (14). For notational simplicity, we omit the dependence of $y, w, \phi$ and $\psi$ on the initial condition $y_{0}$.

Substituting $y(t)$ and $w(t)$ in (14), we obtain

$$
\begin{aligned}
\phi(t)= & \int_{t_{0}}^{t} \psi(s) \mathrm{d} s+A_{u}\left(y_{0}+\int_{t_{0}}^{t} \phi(s) \mathrm{d} s, t\right), \\
\psi(t)= & -\mu \int_{t_{0}}^{t} \psi(s) \mathrm{d} s-M_{u}\left(y_{0}+\int_{t_{0}}^{t} \phi(s) \mathrm{d} s, t\right) \int_{t_{0}}^{t} \psi(s) \mathrm{d} s \\
& -\kappa \mu^{1 / 2} \int_{t_{0}}^{t} \frac{\psi(s)}{\sqrt{t-s}} \mathrm{~d} s+B_{u}\left(y_{0}+\int_{t_{0}}^{t} \phi(s) \mathrm{d} s, t\right) .
\end{aligned}
$$


The right-hand sides of these equations define a mapping $P$ as

$$
(P \Phi)(t)=\left(\begin{array}{c}
\int_{t_{0}}^{t} \psi(s) \mathrm{d} s+A_{u}(y(t), t) \\
-\int_{t_{0}}^{t}\left[\mu+\frac{\kappa \mu^{1 / 2}}{\sqrt{t-s}}+M_{u}(y(t), t)\right] \psi(s) \mathrm{d} s+B_{u}(y(t), t)
\end{array}\right)
$$

where $\Phi=(\phi, \psi) \in \mathbb{R}^{2 n}$ and $y(t)=y_{0}+\int_{t_{0}}^{t} \phi(s) \mathrm{d} s$.

We will show that the mapping $P$ has a unique fixed point in $X_{T, K}$ for some $T, K>0$. Then the existence of the above mentioned solution of (14) follows directly.

The following lemma shows that for an appropriate choice of $T$ and $K, P$ maps $X_{T, K}$ into itself.

Lemma 3. Assume that (H1) holds. Then for $K \geq 4 L_{b}$ and any $y_{0} \in \mathcal{D}$, there exists $\delta>0$ such that, for any $T \in[0, \delta]$, we have $P: X_{T, K} \rightarrow X_{T, K}$.

Proof. The continuity of $P \Phi: \mathbb{R}^{+} \rightarrow \mathbb{R}^{2 n}$ follows from assumption (H1). We also have

$$
\begin{aligned}
|P \Phi(t)| \leq & \left\|\int_{t_{0}}^{t} \psi(s) \mathrm{d} s+A_{u}(y(t), t)\right\|_{\infty} \\
& +\left\|-\int_{t_{0}}^{t}\left[\mu+\frac{\kappa \mu^{1 / 2}}{\sqrt{t-s}}+M_{u}(y(t), t)\right] \psi(s) \mathrm{d} s+B_{u}(y(t), t)\right\|_{\infty} \\
\leq & \|\psi\|_{\infty}\left(\left(t-t_{0}\right)+\mu\left(t-t_{0}\right)+2 \kappa \mu^{1 / 2} \sqrt{t-t_{0}}+L_{b}\left(t-t_{0}\right)\right)+2 L_{b} \\
\leq & \|\Phi\|_{\infty}\left(\left(t-t_{0}\right)+\mu\left(t-t_{0}\right)+2 \kappa \mu^{1 / 2} \sqrt{t-t_{0}}+L_{b}\left(t-t_{0}\right)\right)+2 L_{b} \\
\leq & K\left(\left(t-t_{0}\right)+\mu\left(t-t_{0}\right)+2 \kappa \mu^{1 / 2} \sqrt{t-t_{0}}+L_{b}\left(t-t_{0}\right)\right)+\frac{K}{2}
\end{aligned}
$$

If $\delta>0$ is small enough such that $\delta+\mu \delta+2 \kappa \mu^{1 / 2} \sqrt{\delta}+L_{b} \delta \leq 1 / 2$, we have $\|P \Phi\|_{\infty} \leq K$; and hence $P \Phi \in X_{T, K}$ for any $T \in[0, \delta]$.

We now fix the constant $K=4 L_{b}$ in the following. We show that the map $P$ is a contraction mapping on the space $X_{T, K}$.

Lemma 4. There is $\delta>0$ such that, for any $T \in[0, \delta]$ and $\Phi_{1}, \Phi_{2} \in X_{T, K}$,

$$
\left\|P \Phi_{1}-P \Phi_{2}\right\|_{\infty} \leq \frac{1}{2}\left\|\Phi_{1}-\Phi_{2}\right\|_{\infty}
$$

Proof. Let $\Phi_{1}, \Phi_{2} \in X_{T, K}$ where $\Phi_{i}=\left(\phi_{i}, \psi_{i}\right)^{\top}$. We have

$$
\begin{aligned}
\left|\left(P \Phi_{1}\right)(t)-\left(P \Phi_{2}\right)(t)\right| \leq & \int_{t_{0}}^{t}\left|\psi_{1}(s)-\psi_{2}(s)\right| \mathrm{d} s+\left|A_{u}\left(y_{1}(t), t\right)-A_{u}\left(y_{2}(t), t\right)\right| \\
& +\mu \int_{t_{0}}^{t}\left|\psi_{1}(s)-\psi_{2}(s)\right| \mathrm{d} s \\
& +\kappa \mu^{1 / 2} \int_{t_{0}}^{t} \frac{\left|\psi_{1}(s)-\psi_{2}(s)\right|}{\sqrt{t-s}} \mathrm{~d} s \\
& +\left|M_{u}\left(y_{1}(t), t\right) \int_{t_{0}}^{t} \psi_{1}(s) \mathrm{d} s-M_{u}\left(y_{2}(t), t\right) \int_{t_{0}}^{t} \psi_{2}(s) \mathrm{d} s\right| \\
& +\left|B_{u}\left(y_{1}(t), t\right)-B_{u}\left(y_{2}(t), t\right)\right|
\end{aligned}
$$




$$
\begin{aligned}
\leq & \int_{t_{0}}^{t}\left|\psi_{1}(s)-\psi_{2}(s)\right| \mathrm{d} s+L_{c} \int_{t_{0}}^{t}\left|\phi_{1}(s)-\phi_{2}(s)\right| \mathrm{d} s \\
& +\mu \int_{t_{0}}^{t}\left|\psi_{1}(s)-\psi_{2}(s)\right| \mathrm{d} s \\
& +\kappa \mu^{1 / 2} \int_{t_{0}}^{t} \frac{\left|\psi_{1}(s)-\psi_{2}(s)\right|}{\sqrt{t-s}} \mathrm{~d} s \\
& +L_{b} \int_{t_{0}}^{t}\left|\psi_{1}(s)-\psi_{2}(s)\right| \mathrm{d} s \\
& +L_{c}\left(\left(t-t_{0}\right)|| \psi_{2} \|_{\infty}\right) \int_{t_{0}}^{t}\left|\phi_{1}(s)-\phi_{2}(s)\right| \mathrm{d} s \\
& +L_{c} \int_{t_{0}}^{t}\left|\phi_{1}(s)-\phi_{2}(s)\right| \mathrm{d} s,
\end{aligned}
$$

where we have used the Lipschitz continuity of $A_{u}(\cdot, t), B_{u}(\cdot, t)$ and $M_{u}(\cdot, t)$. We also used the fact that

$$
\begin{aligned}
& \left|M_{u}\left(y_{1}, t\right) \int_{t_{0}}^{t} \psi_{1}(s) \mathrm{d} s-M_{u}\left(y_{2}, t\right) \int_{t_{0}}^{t} \psi_{2}(s) \mathrm{d} s\right|= \\
& \quad\left|M_{u}\left(y_{1}, t\right) \int_{t_{0}}^{t}\left(\psi_{1}(s)-\psi_{2}(s)\right) \mathrm{d} s+\left(M_{u}\left(y_{1}, t\right)-M_{u}\left(y_{2}, t\right)\right) \int_{t_{0}}^{t} \psi_{2}(s) \mathrm{d} s\right| \\
& \leq\left\|M_{u}\left(y_{1}, t\right)\right\| \int_{t_{0}}^{t}\left|\psi_{1}(s)-\psi_{2}(s)\right| \mathrm{d} s+\left\|M_{u}\left(y_{1}, t\right)-M_{u}\left(y_{2}, t\right)\right\| \int_{t_{0}}^{t}\left|\psi_{2}(s)\right| \mathrm{d} s .
\end{aligned}
$$

As a result, we obtain

$$
\begin{aligned}
\left|\left(P \Phi_{1}\right)(t)-\left(P \Phi_{2}\right)(t)\right| \leq & \left(\left(t-t_{0}\right)+\mu\left(t-t_{0}\right)+2 \kappa \mu^{1 / 2} \sqrt{t-t_{0}}+L_{b}\left(t-t_{0}\right)\right)\left\|\psi_{1}-\psi_{2}\right\|_{\infty} \\
& +\left(2 L_{c}\left(t-t_{0}\right)+L_{c} K\left(t-t_{0}\right)^{2}\right)\left\|\phi_{1}-\phi_{2}\right\|_{\infty} .
\end{aligned}
$$

Therefore, one can take $\delta>0$ small enough such that, for any $t \in\left[t_{0}, t_{0}+\delta\right]$,

$$
\begin{aligned}
\left|\left(P \Phi_{1}\right)(t)-\left(P \Phi_{2}\right)(t)\right| & \leq \frac{1}{4}\left(\left\|\phi_{1}-\phi_{2}\right\|_{\infty}+\left\|\psi_{1}-\psi_{2}\right\|_{\infty}\right) \\
& \leq \frac{1}{2}\left\|\Phi_{1}-\Phi_{2}\right\|_{\infty} .
\end{aligned}
$$

Hence we get the contraction property

$$
\left\|P \Phi_{1}-P \Phi_{2}\right\|_{\infty} \leq \frac{1}{2}\left\|\Phi_{1}-\Phi_{2}\right\|_{\infty} .
$$

Since $P$ is a contraction mapping on the complete metric space $X_{T, K}$, it has a unique fixed point in $X_{T, K}$. Therefore, there are unique continuous functions $\phi, \psi:\left[t_{0}, t_{0}+\delta\right] \rightarrow \mathbb{R}^{n}$ such that the functions $y, w$ defined by (15) solve the MR equation (14) and satisfy $y\left(t_{0}\right)=y_{0}$ and $w\left(t_{0}\right)=0$. This concludes the proof of Theorem 2.

\section{References}

[1] C. M. Tchen. Mean value and correlation problems connected with the motion of small particles suspended in a turbulent fluid. PhD thesis, TU Delft, 1947. 
[2] S. Corrsin and J. Lumley. On the equation of motion for a particle in turbulent fluid. App. Sci. Res., Section A, 6(2-3):114-116, 1956.

[3] M. R. Maxey and J. J. Riley. Equation of motion for a small rigid sphere in a nonuniform flow. Phys. Fluids, 26:883-889, 1983.

[4] E. van Sebille, M. H. England, and Gary Froyland. Origin, dynamics and evolution of ocean garbage patches from observed surface drifters. Env. Res. Lett., 7(4):044040, 2012.

[5] D. Vallero. Fundamentals of air pollution. Academic press, fourth edition, 2008.

[6] J. V. Boussinesq. Sur la résistance qu'oppose un fluide indéfini au repos, sans pesanteur, au mouvement varié d'une sphére solide qu'il mouille sur toute sa surface, quand les vitesses restent bien continues et assez faibles pour que leurs carrés et produits soient négligeables. Comptes Rendu de l'Academie des Sciences, 100:935-937, 1885.

[7] A. B. Basset. A treatise on hydrodynamics. Deighton, Bell and Co, Cambridge, 1888.

[8] C. W. Oseen. Hydrodynamik. Akademische Verlagsgesellschaft, Leipzig, 1927.

[9] T. R. Auton, J. C. R. Hunt, and M. Prud'Homme. The force exerted on a body in inviscid unsteady non-uniform rotational flow. J. of Fluid Mech., 197:241-257, 1988.

[10] M. R. Maxey. The equation of motion for a small rigid sphere in a nonuniform or unsteady flow. In Gas-solid flows, 1993, volume 166, pages 57-62. The American society of mechanical engineers, 1993.

[11] I. Podlubny. Fractional Differential Equations. An Introduction to Fractional Derivatives, Fractional Differential Equations, Some Methods of Their Solution and Some of Their Applications. Academic Press, San Diego - New York - London, 1999.

[12] A. Daitche. Advection of inertial particles in the presence of the history force: Higher order numerical schemes. Journal of Computational Physics, 254:93-106, 2013.

[13] A. B. Basset. An elementary treatise on hydrodynamics and sound. Cambridge: Deighton, Bell and Co, 1890 .

[14] M. R. Maxey. The gravitational settling of aerosol particles in homogeneous turbulence and random flow fields. J. Fluid Mech., 174(1):441-465, 1987.

[15] A. Babiano, J. H. E. Cartwright, O. Piro, and A. Provenzale. Dynamics of a small neutrally buoyant sphere in a fluid and targeting in Hamiltonian systems. Phys. Rev. Lett., 84:5764-5767, 2000 .

[16] G. Haller and T. Sapsis. Where do inertial particles go in fluid flows? Physica D, 237(5): 573-583, 2008.

[17] F. Candelier, J. R. Angilella, and M. Souhar. On the effect of the Boussinesq-Basset force on the radial migration of a Stokes particle in a vortex. Physics of Fluids, 16(5):1765-1776, 2004.

[18] R. Toegel, S. Luther, and D. Lohse. Viscosity destabilizes sonoluminescing bubbles. Phys. Rev. Lett., 96(11):114301, 2006.

[19] V. Garbin, B. Dollet, M. Overvelde, D. Cojoc, E. Di Fabrizio, L. van Wijngaarden, A. Prosperetti, N. de Jong, D. Lohse, and M. Versluis. History force on coated microbubbles propelled by ultrasound. Phys. Fluids, 21(9), 2009.

[20] A. Daitche and T. Tél. Memory effects are relevant for chaotic advection of inertial particles. Phys. Rev. Lett., 107:244501, 2011. 
[21] K. Guseva, U. Feudel, and T. Tél. Influence of the history force on inertial particle advection: Gravitational effects and horizontal diffusion. Phys. Rev. E, 88(4):042909, 2013.

[22] A. Daitche and T. Tél. Memory effects in chaotic advection of inertial particles. New J. of Phys., 16(7):073008, 2014.

[23] C. M. F. Coimbra and R. H. Rangel. General solution of the particle momentum equation in unsteady Stokes flows. J. of Fluid Mech., 370(1):53-72, 1998.

[24] T. A. Burton and I. K. Purnaras. Singular integro-differential equations with small kernels. J. of Integral Equations and Applications, 25(1):1-20, 2013.

[25] T. A. Burton and I. K. Purnaras. $L^{p}$-solutions of singular integro-differential equations. J. of Math. Anal. Appl., 386(2):830-841, 2012.

[26] I. Petrás. Fractional-order nonlinear systems: modeling, analysis and simulation. Springer, 2011.

[27] T. Sapsis and G. Haller. Inertial particle dynamics in a hurricane. J. of the Atmos. Sci., 66(8): 2481-2492, 2009 .

[28] T. P. Sapsis, N. T. Ouellette, J. P. Gollub, and G. Haller. Neutrally buoyant particle dynamics in fluid flows: Comparison of experiments with Lagrangian stochastic models. Phys. Fluids, 23 (9):093304, 2011. 